\title{
Hábitos alimenticios de la ardilla Sciurus variegatoides (Rodentia: Sciuridae) en la Península de Nicoya, Costa Rica
}

\author{
Javier Monge ${ }^{1} \&$ Luko Hilje $^{2}$ \\ 1 Centro de Investigaciones en Protección de Cultivos, Universidad de Costa Rica, San José, Costa Rica; \\ jimonge@cariari.ucr.ac.cr \\ 2 Unidad de Fitoprotección, Centro Agronómico Tropical de Investigación y Enseñanza, Turrialba (CATIE), Costa Rica; \\ lhilje@catie.ac.cr
}

Recibido 05-XII-2003. Corregido 02-V-2005. Aceptado 16-VIII-2005.

\begin{abstract}
Feeding habits of the squirrel Sciurus variegatoides (Rodentia: Sciuridae) in the Nicoya Peninsula, Costa Rica. Food items consumed by the squirrel Sciurus variegatoides atrirufus were determined in an agricultural setting in the Nicoya Península (947' N, $84^{\circ} 56^{\prime} \mathrm{W}$ ), Costa Rica, where two life zones (Premontane Moist Forest Basal Belt Transition, and Tropical Dry Forest) predominate. By analyzing the gut contents of 120 squirrels, from February 1987 through January 1988, it was determined that coconut (Cocos nucifera), indian almond (Terminalia catappa) and flamboyant (Delonix regia) were the most common dietary items. There were differences in food consumption according to age: adults preferred coconut, whereas young individuals preferred almond. This finding can be explained in terms of fruit characteristics, as well as tree architecture and accessibility for squirrels; almendro trees provide higher protection and a more accessible food resource, so that it was better used by young individuals. Rev. Biol. Trop. 54(2): 681-686. Epub 2006 Jun 01.
\end{abstract}

Key words: squirrel, Sciurus variegatoides atrirufus, food habits, Costa Rica.

La ardilla Sciurus variegatoides (Ogilby 1839) se distribuye desde el sur de Chiapas, México hasta el centro de Panamá (Hall 1981) y se encuentra en diferentes ambientes naturales y alterados desde el nivel del mar hasta los 2500 de elevación (Best 1995). A pesar de su amplia distribución en América Central y a que es abundante en sitios donde no es cazada (Reid 1997), esta especie ha sido relativamente poco estudiada.

Un estudio acerca de su dieta fue hecho por Glanz (1984) en su ambiente natural en Panamá, mientras que otros estudios realizados en Costa Rica hacen referencia a la especie sin ser específicos para ésta (Janzen 1972, 1982a, 1982b; Boucher 1981; Bradford y Smith 1977), salvo la observación relacionada con el consumo de hongos realizada por Gómez (1983). Algunas observaciones de otros aspectos de su historia natural han sido resumidas por Best (1995). Se le considera una especie dañina dado que afecta diversos cultivos como: banano (Musa x acuminata), papaya (Carica papaya), coco (Cocos nucifera), aguacate (Persea americana), mango (Mangifera indica), chayote (Sechium edule), zanahoria (Daucus carota), arveja (Pisum sativum) y macadamia (Macadamia integrifolia) y especies forestales como pochote (Bombacopsis quinata) y eucalipto (Eucalyptus spp.) (Hilje y Monge 1988).

El objetivo de este estudio fue determinar los hábitos alimenticios de la ardilla ( $S$. variegatoides atrirufus) en un área agrícola de la Península de Nicoya, Costa Rica. 


\section{MATERIALES Y MÉTODOS}

Área de estudio. El estudio se efectuó en la Hacienda Curú, en el cantón Paquera, provincia de Puntarenas, ubicada en la región suroeste de la Península de Nicoya, Costa Rica $\left(9^{\circ} 47^{\prime} \mathrm{N}, 8^{\circ} 56^{\prime} \mathrm{W}\right)$. La precipitación media anual es de $1957.43 \mathrm{~mm}$, con una época seca (precipitación mensual inferior a $50 \mathrm{~mm}$ ) que abarca desde diciembre hasta abril, y una época lluviosa de mayo a noviembre, siendo los meses de setiembre y octubre los más lluviosos con precipitaciones mensuales superiores a 300 mm (Anónimo 1987). La temperatura promedio mensual varía entre $25-30^{\circ} \mathrm{C}$ (Anónimo 1985). En el sitio se encuentran las zonas de vida bosque húmedo premontano transición a basal y bosque seco tropical (Tosi 1969).

En la época de este estudio, el uso de la tierra en Hacienda Curú estaba representado por agricultura (78 ha), ganadería (371 ha) y bosques y manglares (667 ha). Los cultivos agrícolas fueron: coco (41\%), mango (11\%), palma africana $(8 \%)$, papaya $(7 \%)$ y otros en menor escala como guanábana (Annona muricata) y aguacate. Un 30\% del área agrícola se usó para cultivos de ciclo corto, como melón (Cucurbita melo) y maíz (Zea mays) (Bustos et al. 1986).

Metodología: De febrero de 1987 a enero de 1988 se recolectaron 10 especímenes por mes, provenientes principalmente de áreas agrícolas, potreros arbolados o cercas vivas que bordeaban áreas de potrero. No se incluyeron individuos que habitaban en bosques. Los especímenes de referencia se encuentran en la colección del Departamento de Historia Natural del Museo Nacional de Costa Rica (MNCR 763-MNCR 778).

La mayoría de las recolecciones se efectuaron por la mañana, entre las 6 y 10 am, e incluyeron individuos de ambos sexos y de varias clases de edad (adultos y jóvenes). Los especímenes se pesaron y midieron, asimismo se evaluaron algunas características externas (pelaje y genitales) e internas (aparato reproductivo), las cuales fueron útiles para clasificar cada individuo según su sexo y edad (Monge 1989).

A través de una incisión abdominal se extrajo el tracto digestivo (estómago e intestinos), el cual fue pesado y preservado en alcohol de $70^{\circ}$ o FAA (formalina 5\%, ácido acético 5\% y alcohol 90\%) para su posterior análisis. Para determinar la dieta se analizó el contenido estomacal, observándose si estaba constituido por uno o más componentes y luego a través del análisis visual o con ayuda del estereoscopio se identificaron los componentes alimentarios. La identificación de cada componente fue posible por comparación de semillas, características de la pulpa, tipo de fibras, color de la cáscara o pulpa de frutos de especies conocidas recolectadas en el campo. Para determinar diferencias en la dieta según la edad de los individuos se usó la prueba de diferencias de proporciones (Walpole y Myers 1978):

$$
z=\frac{\hat{p}_{1} \hat{p}_{2}}{\sqrt{\frac{p_{1} q_{1}}{n_{1}}+\frac{p_{2} q_{2}}{n_{2}}}}
$$

\section{RESULTADOS}

Los componentes más importantes de la dieta de $S$. variegatoides fueron coco, almendro (Terminalia catappa) y malinche (Delonix regia), en el Cuadro 1 se puede observar la frecuencia mensual de presencia de los alimentos en el contenido estomacal de los individuos. El coco y el almendro fueron alimentos utilizados durante casi todo el año, mientras que las semillas de malinche se utilizaron en la época seca y principios de la época lluviosa, dada su disponibilidad estacional. Para otros componentes no se pudo definir una época determinada de consumo, por su baja frecuencia de aparición.

El coco y el almendro mostraron su importancia como componente único o combinado, ya que el coco apareció como componente único en 30 ocasiones, en tres junto con almendro 
CUADRO 1

Frecuencia mensual de presencia de los alimentos en el contenido estomacal de S. v. atrirufus recolectados. Hacienda Curú, Puntarenas. Período febrero 1987-enero 1988

\begin{tabular}{|c|c|c|c|c|c|c|c|c|c|c|c|c|c|c|}
\hline & & & & & & & & & & & & & & \\
\hline Componentes & $\mathrm{F}$ & M & A & M & $\mathrm{J}$ & $\mathrm{J}$ & A & $\mathrm{S}$ & $\mathrm{O}$ & $\mathrm{N}$ & $\mathrm{D}$ & $\mathrm{E}$ & Total & Porc. \\
\hline Coco Cocos nucifera & 3 & 6 & 2 & 0 & 3 & 4 & 2 & 2 & 7 & 4 & 2 & 7 & 42 & 35.00 \\
\hline $\begin{array}{l}\text { Almendro } \\
\text { Terminalia catappa }\end{array}$ & 2 & 3 & 0 & 5 & 7 & 2 & 1 & 4 & 4 & 5 & 0 & 1 & 34 & 28.33 \\
\hline Malinche Delonix regia & 0 & 1 & 1 & 6 & 1 & 1 & 0 & 0 & 0 & 0 & 0 & 2 & 12 & 10.00 \\
\hline Guayaba Psidium guajava & 0 & 1 & 1 & 0 & 0 & 0 & 0 & 0 & 0 & 0 & 0 & 0 & 2 & 1.67 \\
\hline $\begin{array}{l}\text { Guácimo } \\
\text { Guazuma ulmifolia }\end{array}$ & 0 & 0 & 0 & 1 & 0 & 0 & 0 & 0 & 0 & 0 & 0 & 0 & 1 & 0.83 \\
\hline $\begin{array}{l}\text { Palma real } \\
\text { Attalea butyracea }\end{array}$ & 0 & 0 & 0 & 0 & 1 & 0 & 3 & 1 & 0 & 0 & 1 & 0 & 6 & 5.00 \\
\hline Mango Mangifera indica & 0 & 0 & 0 & 0 & 0 & 1 & 0 & 0 & 0 & 2 & 0 & 0 & 3 & 2.50 \\
\hline Sin identificar & 1 & 0 & 5 & 0 & 0 & 3 & 6 & 1 & 2 & 2 & 2 & 1 & 23 & 19.17 \\
\hline Sin información & 5 & 0 & 2 & 0 & 0 & 0 & 0 & 3 & 0 & 0 & 7 & 0 & 17 & 17.17 \\
\hline
\end{tabular}

y en cuatro junto con la palma real (Attalea butyracea), mientras que el almendro apareció como componente único en 25 ocasiones, en tres con coco y en cuatro con malinche.

En cuanto al peso del tracto digestivo, representado principalmente por el contenido estomacal, fue de $54.44 \pm 25.11 \mathrm{~g}(\mathrm{n}=30)$ para el coco; de $44.37 \pm 13.50 \mathrm{~g}(\mathrm{n}=25)$ para el almendro y de $42.40 \pm 12.27 \mathrm{~g}(\mathrm{n}=6)$ para el malinche. Estos pesos fueron superados por los contenidos estomacales compuestos por al menos dos componentes, como el almendro-malinche con $66.30 \pm 9.47 \mathrm{~g}(\mathrm{n}=4)$, coco-palma real $59.05 \pm 9.97(\mathrm{n}=4)$ y cocoalmendro $55.17 \pm 7.52 \mathrm{~g}(\mathrm{n}=3)$.

Los alimentos no fueron igualmente consumidos por los individuos según su edad. El coco se constituyó en el alimento más consumido por los adultos, existiendo una diferencia estadística significativa en la proporción de individuos en que se identificó este alimento en el contenido estomacal con respecto a la proporción de los otros componentes alimenticios (Cuadro 2). Los machos adultos utilizaron el coco como componente único o en forma conjunta con otro alimento en 22 ocasiones, para un $41.5 \%$ del total de adultos recolectados, mientras que el almendro fue consumido en nueve ocasiones (17\%). El coco también apareció en el contenido estomacal de 12 hembras adultas recolectadas $(40 \%)$, seguido por el almendro con seis ocasiones (20\%). Para los jóvenes, el alimento más consumido fue el almendro (Cuadro 3), que se encontró en cuatro contenidos estomacales de machos jóvenes ( $21 \%$ del total de machos jóvenes recolectados) y en seis contenidos estomacales de hembras jóvenes (33.3\% del total de hembras jóvenes).

\section{DISCUSIÓN}

El análisis del contenido estomacal de 120 ardillas indicó que el coco fue el alimento más importante, lo cual pudo deberse a que fue el cultivo dominante del área agrícola de recolección de especímenes (Bustos et al. 1986); sin embargo, también puede deberse por el valor nutritivo de este componente alimenticio (FEDNA 2003). A su vez, las características propias del fruto de coco pudieron haber influido en la inclusión a la muestra de estudio 
CUADRO 2

Probabilidades de Z para las pruebas de diferencia entre las distintas proporciones de los diferentes componentes en el contenido estomacal que presentaron individuos adultos de $\mathrm{S}$. v. atrirufus

$\begin{array}{lccccccc}\text { Componente } & & \text { Coco } & \text { Almendro } & \text { Malinche } & \text { Palma real } & \text { Guayaba } & \text { Guácimo } \\ & \text { Proporción } & 0.42 & 0.28 & 0.11 & 0.05 & 0.02 & 0.01 \\ \text { Coco } & 0.42 & & 0.024 & 0.000 & 0.000 & 0.000 & 0.000 \\ \text { Almendro } & 0.28 & & & 0.002 & 0.000 & 0.000 & 0.000 \\ \text { Malinche } & 0.11 & & & 0.073 & 0.013 & 0.004 \\ \text { Palma real } & 0.05 & & & & & 0.202 & 0.085 \\ \text { Guayaba } & 0.02 & & & & & & 0.280 \\ \text { Guácimo } & 0.01 & & & & & & \end{array}$

CUADRO 3

Probabilidades de Z para las pruebas de diferencia entre las distintas proporciones de los diferentes componentes en el contenido estomacal que presentaron individuos juveniles de S. v. atrirufus

\begin{tabular}{lcccccc}
\multicolumn{1}{c}{ Componente } & & Almendro & Coco & Mango & Malinche & Palma real \\
& Proporción & 0.324 & 0.162 & 0.108 & 0.081 & 0.054 \\
Almendro & 0.324 & & 0.049 & 0.010 & 0.003 & 0.001 \\
Coco & 0.162 & & & 0.248 & 0.141 & 0.064 \\
Mango & 0.108 & & & & 0.345 & 0.196 \\
Malinche & 0.081 & & & & 0.321 \\
Palma real & 0.054 & & & & &
\end{tabular}

de ardillas que estuvieran consumiendo este alimento, ya que la dureza de la cáscara del fruto delataba la presencia de ardillas más que cuando consumía otros posibles alimentos. Debe tenerse en cuenta que para hacer uso del coco (endospermo del fruto) requiere una labor de aproximadamente tres días, lo cual aumenta la probabilidad de ser encontrado consumiendo este alimento.

En el caso del almendro, su importancia como componente de la dieta de la población de ardillas estudiadas puede deberse a la alta disponibilidad de este alimento durante la época de fructificación, y además por las características mismas del fruto y arquitectura del árbol, lo cual ofrece condiciones como fuente de alimento y refugio principalmente para los individuos jóvenes. Al comparar las características del fruto de almendro en cuanto a tamaño y dureza, la cobertura que ofrece el follaje y el acceso al árbol mismo, es comprensible la preferencia de este alimento más que por el coco, por parte de los jóvenes.

Otros alimentos como el mango y el guácimo (Guazuma ulmifolia) estuvieron poco representados en este estudio, lo cual contrasta con las observaciones de Glanz (1984) dado que éstos junto con el jobo (Spondias mombin) representaron un 49.4\% de su dieta en Panamá. El jobo estuvo presente en las zonas boscosas (Bustos et al. 1986) en las cercanías al área de recolección, y dado que no se tomaron especímenes provenientes de áreas boscosas, se puede explicar por qué este alimento no estuvo representado en el presente estudio. Una situación similar pudo presentarse con Sterculia 
apetala, cuyas semillas son consumidas por S. variegatoides (Janzen 1972), la cual está presente en las zonas boscosas cercanas al área de recolección, pero no incluidas dentro de ésta.

En cuanto al guácimo, Janzen (1982a) hace referencia a que $S$. variegatoides ha sido observada consumiendo sus frutos, aunque desconocía si ingería las semillas, mientras que Glanz (1984) indica que los componentes utilizados de esta especie fueron suaves (frutos suaves y flores), lo cual puede haber influido para no ser reconocido con mayor frecuencia con la metodología seguida en este estudio. Este criterio no es aplicable al mango, que aún cuando el fruto maduro es suave, por las características de la pulpa es fácilmente reconocible. Esta limitante metodológica pudo haber sido más influyente en el grupo de los jóvenes, ya que una porción de contenidos estomacales no pudo ser identificado, lo cual obedece a que hacen uso de partes más suaves (hojas, rebrotes, flores) de diversas especies, lo que dificulta su identificación con respecto a las posibilidades que ofrece alimentos más duros como frutos y semillas.

La palma real tuvo poca representatividad en la dieta de la ardilla, cuyos frutos fueron consumidos en la época lluviosa. Bradford y Smith (1977) sugirieron que $S$. variegatoides es probablemente uno de los roedores depredadores de semillas de Scheelea (=Attalea). Por su parte, Glanz (1984) observó en marzo y junio-julio a $S$. variegatoides alimentándose de $S$. zoonensis (=A. butyracea), aunque estas palmas no representaron un alimento frecuente para esta especie en su estudio, situación que coincide con el presente estudio y también con las observaciones de Fleming (1970), quien observó en solo una ocasión a $S$. variegatoides llevando una nuez de Attalea.

Otra especie que ha formado parte de la dieta de $S$. variegatoides en Costa Rica es el jícaro (Crescentia alata), pero en raras ocasiones puede roer los frutos maduros (Janzen 1982b), lo cual no se observó en el presente estudio, a pesar de la presencia de unos pocos árboles de esta especie dentro del área de estudio. Con respecto al roble (Quercus oleoides),
Boucher (1981) observó a $S$. variegatoides comiendo bellotas de esta especie, la cual no estuvo representada en el área de estudio.

Puede concluirse que $S$. variegatoides tiene una dieta variada, compuesta por más de 25 especies vegetales, además de hongos, y considerando la diversidad de ambientes existentes en su ámbito de distribución, es posible que esta lista aumente con estudios posteriores en otras localidades. En términos generales, se considera una especie oportunista, que hace uso de diferentes componentes en función de su disponibilidad, situación que la ha llevado a ser considerada como una especie plaga, principalmente cuando habita en áreas agrícolas y forestales, comiendo frutos de banano, papaya, mango, maíz y aguacate (McPherson 1985). La información suministrada por el presente estudio, complementada con la de otros estudios referidos, permiten disponer información básica acerca de uno de los aspectos de su historia natural de gran importancia para fines de manejo.

\section{AGRADECIMIENTOS}

AlaUniversidad Nacionaly la Organización para Estudios Tropicales, por el financiamiento parcial de la investigación. A la familia Shutt Valle, por facilitar su propiedad para el estudio. A Juan Ramón Navarro (Escuela de Agronomía, Universidad de Costa Rica) por su apoyo en el análisis estadístico. A los revisores anónimos, por sus valiosas sugerencias al manuscrito original.

\section{RESUMEN}

Se determinaron los componentes de la dieta de la ardilla Sciurus variegatoides atrirufus en un área agrícola de la Península de Nicoya, Costa Rica $\left(9^{\circ} 47^{\prime} \mathrm{N}, 84^{\circ} 56^{\prime}\right.$ W), correspondiente a las zonas de vida Bosque Húmedo Premontano Transición a Basal y Bosque Seco Tropical. Al analizar el contenido estomacal de 120 ardillas, entre febrero de 1987 y enero de 1988, se determinó que los alimentos más importantes fueron el coco (Cocos nucifera), 
el almendro (Terminalia catappa) y el malinche (Delonix regia). Hubo diferencias en el tipo de alimento según la edad. Los adultos consumieron más el coco y los jóvenes el almendro, lo cual se explica por las características del fruto, así como por la arquitectura y acceso al árbol; el almendro aporta mejores condiciones de protección y un recurso alimenticio más accesible, por lo que fue mejor aprovechado por los individuos jóvenes.

Palabras clave: ardilla, Sciurus variegatoides atrirufus, hábitos alimenticios, Costa Rica.

\section{REFERENCIAS}

Anónimo. 1985. Atlas climatológico de Costa Rica. Ministerio de Agricultura y Ganadería. San José, Costa Rica

Anónimo. 1987. Registros de precipitación de la Estación Meteorológica de Paquera, Puntarenas. Instituto Meteorológico Nacional. San José, Costa Rica.

Best, T.L. 1995. Sciurus variegatoides. Mammalian Species 500: $1-6$.

Bradford, D.F. \& C.C. Smith. 1977. Seed predation and seed number in Scheelea palm fruits. Ecology 58: 667-673.

Boucher, D.H. 1981. Seed predation by mammals and forest dominance by Quercus oleoides, a tropical lowland oak. Oecologia 49: 409-414.

Bustos, N. et al. 1986. Plan de Manejo de la Hacienda Curú, Paquera, Puntarenas. Escuela de Ciencias Ambientales, Universidad Nacional, Heredia, Costa Rica.

Fundación Española para el Desarrollo de la Nutrición Animal (FEDNA). 2003. Tablas FEDNA de composición y valor nutritivo de alimentos para la formulación de piensos compuestos. (También disponible en línea, 16 de mayo del 2005, http://www.etsia.upm. es/fedna/grasasyaceites/aceite_coco.htm).

Fleming, T.H. 1970. Notes on the rodent faunas of two Panamanian forests. J. Mamm. 51: 473-490.
Glanz, W.E. 1984. Food and habitat use by two sympatric Sciurus species in Panama. J. Mamm. 65: 342-347.

Gómez, L.D. 1983. Variegated squirrels eat fungi, too. Brenesia 21: 458-459.

Hall, E.R. 1981. The mammals of North America. Wiley, Nueva York. 606 p +90 p.

Hilje, L. \& J. Monge, 1988. Lista preliminar y consideraciones generales acerca de los animales vertebrados plaga en Costa Rica. Manejo Integrado de Plagas 10: $39-52$.

Janzen, D. 1972. Escape in space by Sterculia apetala seeds from the bug Dysdercus fasciatus in a Costa Rican deciduous forest. Ecology 53: 350-361.

Janzen, D. 1982a. Natural history of guacimo fruits (Sterculiaceae: Guazuma ulmifolia) with respect to consumption by large mammals. Amer. J. Bot. 69: 1240-1250.

Janzen, D. 1982b. Fruit traits, and seed consumption by rodents, of Crecentia alata (Bignoniaceae) in Santa Rosa National Park, Costa Rica. Amer. J. Bot. 69: 1258-1268.

McPherson, A.B. 1985. A biogeographical analysis of factors influencing the distribution of Costa Rican rodents. Brenesia 23: 97-273.

Monge M., J. 1989. Ciclo reproductivo y dieta de la ardilla, Sciurus variegatoides (Sciuridae: Rodentia) en la Península de Nicoya, Costa Rica. Tesis de Licenciatura. Escuela de Ciencias Ambientales, Universidad Nacional, Heredia, Costa Rica. 81 p.

Reid, F.A. 1997. A field guide to the mammals of Central America and Southeast México. Oxford, Nueva York. $334 \mathrm{p}$.

Tosi, J. 1969. Mapa ecológico de Costa Rica. Escala 1:750 000. Centro Científico Tropical. San José, Costa Rica.

Walpole, R.E. \& R.H. Myers. 1978. Probability and statistics for engineers and scientists. Macmillan, Nueva York. 580 p. 